\title{
Finitely Generated Modules over Group Rings of a Direct Product of Two Cyclic Groups
}

\author{
Ahmed Najim and Mohammed Elhassani Charkani \\ Department of Mathematics and Informatics, Faculty of Science Dhar Mahraz, Sidi Mohamed Ben Abdellah University, \\ $30000 \mathrm{Fez}$, Morocco \\ Correspondence should be addressed to Ahmed Najim; najim.sefrou@gmail.com
}

Received 27 August 2014; Accepted 15 November 2014; Published 1 December 2014

Academic Editor: Zhongshan Li

Copyright (C) 2014 A. Najim and M. E. Charkani. This is an open access article distributed under the Creative Commons Attribution License, which permits unrestricted use, distribution, and reproduction in any medium, provided the original work is properly cited.

Let $K$ be a commutative field of characteristic $p>0$ and let $G=G_{1} \times G_{2}$, where $G_{1}$ and $G_{2}$ are two finite cyclic groups. We give some structure results of finitely generated $K[G]$-modules in the case where the order of $G$ is divisible by $p$. Extensions of modules are also investigated. Based on these extensions and in the same previous case, we show that $K[G]$-modules satisfying some conditions have a fairly simple form.

\section{Introduction}

Let $K$ be a field of characteristic $p>0$ and let $G$ be a finite group. The study of $K[G]$-modules in the case where the order of $G$ is divisible by $p$ is a very difficult task. When $G$ is a finite abelian $p$-group, we find in [1] the following statement: a complete classification of finitely generated $K[G]$-modules is available only when $G$ is cyclic or equal to $C_{2} \times C_{2}$, where $C_{2}$ is the cyclic group of order 2. In [2] we find this classification in these two cases. Still more, in the case where the Sylow $p$ subgroup $P$ of $G$ is not cyclic, the groups $G$ such that $p=2$ and $P$ is dihedral, semidihedral, or generalized quaternion are the only groups for which we can (in principle) classify the indecomposable $K[G]$-modules (see [2]). These reasons just cited show the importance of the study of $K[G]$-modules when $G$ is of order divisible by $p$ and equal to a direct product of two cyclic groups.

Now, let $K$ be a commutative field of characteristic $p>0$ and let $G=G_{1} \times G_{2}$, where $G_{1}$ and $G_{2}$ are two finite cyclic groups. Let $M$ be a finitely generated $K[G]$-module. When $M$ is considered as a module over a subalgebra $K[H]$ of $K[G]$ for a subgroup $H$ of the group $G$, we write $M \downarrow_{H}$.

In Section 2, we show that if $G_{1}$ is a cyclic p-group and the characteristic of $K$ does not divide the order of $G_{2}$, then we can have a complete system of indecomposable pairwise nonisomorphic $K[G]$-modules. In the rest, we assume that
$G_{1}=\left\langle\sigma_{1}\right\rangle$ and $G_{2}=\left\langle\sigma_{2}\right\rangle$ are cyclic p-groups. Under conditions that $M \downarrow_{G_{1}}$ is a free $K\left[G_{1}\right]$-module and that $M /\left(\sigma_{1}-1\right) M$ is a free $K\left[G_{2}\right]$-module, we show that $M$ is a free $K[G]$-module. We also show that if $\sigma_{2}$ is of order $p^{n}$, $n \neq 0$, and $H_{2}$ is the subgroup of $G_{2}$ generated by $\sigma_{2}^{p^{n-r}}$ with $0<r \leq n$, then under certain conditions $M$ is a free $K\left[G_{1} \times H_{2}\right]$-module. The fact that $M \downarrow_{G_{1}}$ must be a free $K\left[G_{1}\right]$ module is one of these conditions, and exactly in the end of this section we give a result that shows when this condition is satisfied. In Section 3 and always in the case where $G_{1}$ and $G_{2}$ are cyclic p-groups, we show that under some conditions $K[G]$-modules have a fairly simple form. But in case $p=2$, $G_{1}$ and $G_{2}$ are two cyclic groups of respective orders 2 and $2^{n}$, $n \neq 0$; these modules have this simple form without any other assumptions other than that they must be finitely generated over $K[G]$.

\section{Free $K\left[C_{p^{m}} \times C_{p^{n}}\right]$-Modules of Finite Rank}

Throughout this paper, rings are assumed to be commutative with unity. We begin this section by giving a weak version of Nakayama's lemma with an elementary proof.

Lemma 1 (Nakayama). Let $G$ be a p-group with $p$ odd, $R$ a ring of characteristic $p^{k}$ where $k$ is a natural number, 
$M$ an $R[G]$-module (not necessarily finitely generated), and $N$ a submodule of $M$ and $\sigma \in G$. Then, one has the following:

(1) if $(\sigma-1) M=M$, then $M=0$;

(2) if $M=(\sigma-1) M+N$, then $M=N$;

(3) if $x_{i}, i \in I$, are representatives in $M$ of a generating family of $M /(\sigma-1) M$, then $\left(x_{i}\right)_{i \in I}$ generate $M$.

Proof. (1) Let $p^{r}$ be the order of $G$. We have

$$
\begin{aligned}
(\sigma-1)^{p^{r}} & =\sum_{i=0}^{p^{r}} C_{p^{r}}^{i}(-1)^{i} \sigma^{p^{r}-i} \\
& =\sigma^{p^{r}}+(-1)^{p^{r}}+\sum_{i=1}^{p^{r}-1} C_{p^{r}}^{i}(-1)^{i} \sigma^{p^{r}-i} \\
& =\sum_{i=1}^{p^{r}-1} C_{p^{r}}^{i}(-1)^{i} \sigma^{p^{r}-i} \\
& =p \sum_{i=1}^{p^{r}-1} \frac{1}{p} C_{p^{r}}^{i}(-1)^{i} \sigma^{p^{r}-i} .
\end{aligned}
$$

For $1 \leq i \leq p^{r}-1, p \mid C_{p^{r}}^{i}$, so $(1 / p) C_{p^{r}}^{i}$ is a natural number. So

$$
\begin{aligned}
(\sigma-1)^{k p^{r}} & =\left((\sigma-1)^{p^{r}}\right)^{k} \\
& =\left(p \sum_{i=1}^{p^{r}-1} \frac{1}{p} C_{p^{r}}^{i}(-1)^{i} \sigma^{p^{r}-i}\right)^{k} \\
& =p^{k}\left(\sum_{i=1}^{p^{r}-1} \frac{1}{p} C_{p^{r}}^{i}(-1)^{i} \sigma^{p^{r}-i}\right)^{k} \\
& =0,
\end{aligned}
$$

since $R$ has characteristic $p^{k}$. Now $M=(\sigma-1) M=(\sigma-$ $1)^{2} M=\cdots=(\sigma-1)^{k p^{r}} M=0$.

(2) If $M=(\sigma-1) M+N$, then $M / N=((\sigma-1) M+N) / N=$ $(\sigma-1)(M / N)$, and then, by $(1), M / N=0$ and then $M=N$.

(3) If $N$ is the submodule generated by $x_{i}$, then $M=(\sigma-$ 1) $M+N$, and then by (2) we have $M=N$.

Remark 2. Lemma 1 remains true if $p=2$ and $R$ is of characteristic 2 .

For a ring $R$ of prime characteristic $p$ and for a cyclic group $G$ of order $p^{r}$ generated by an element $\sigma$, we have the following lemma.

Lemma 3. Let $k=p^{r^{\prime}}$ with $0<r^{\prime} \leq r$ and let $H$ be a subgroup of $G$ generated by $\sigma^{p^{r} / k}$. Then, one has $R[G] /(\sigma-1)^{k} R[G] \cong$ $R[H]$ (as R-algebras).
Proof. Define

$$
\begin{gathered}
\psi: \frac{R[G]}{(\sigma-1)^{k} R[G]} \longrightarrow R[H], \\
\bar{\sigma} \longmapsto \sigma^{p^{r} / k},
\end{gathered}
$$

where $\psi$ is a well-defined $R$-algebra homomorphism. It is easy to see that $\psi$ is surjective. As $R[G] /(\sigma-1)^{k} R[G]$ and $R[H]$ are finite free modules of the same rank $k$ over $R, \psi$ is an isomorphism.

Remark 4. With the notation of Lemma 3, $H$ is simply the subgroup of $G$ generated by $\sigma^{p^{r-r^{\prime}}}$.

Let $K$ be a commutative field of characteristic $p>0$ and let $G$ be a direct product of two finite groups $G_{1}$ and $G_{2}$. We have $K[G]=K\left[G_{1} \times G_{2}\right] \cong R\left[G_{2}\right]$, where $R=K\left[G_{1}\right]$.

Assume that $G_{1}$ is a cyclic group of order $p^{m}$ generated by $\sigma_{1}$ and $p$ does not divide the order of $G_{2} . R=K\left[G_{1}\right]$ is a principal Artinian local ring. Indeed $K\left[G_{1}\right] \cong K[X] /(X-$ $1)^{p^{m}}$; this isomorphism is induced by the homomorphism $\Psi: K[X] \rightarrow K[G]$ defined by $\Psi(X)=\sigma_{1} . K[X] /(X-1)^{p^{m}}$ is a principal Artinian local ring with residue field $K$ (up to isomorphism) whose maximal ideal is generated by $\overline{X-1}$. So $R$ is a principal Artinian local ring with residue field $K$ (up to isomorphism) whose maximal ideal is generated by $\sigma_{1}-1$. We have $K[G] \cong R\left[G_{2}\right]$, where $R$ is a principal Artinian local ring of residue field $K$. The characteristic of $K$ does not divide the order of $G_{2}$. Under these conditions, we can apply [3, Theorem 3.6] to have a complete system of indecomposable pairwise nonisomorphic $K[G]$-modules.

In the remainder of this section, we assume that $G_{1}=C_{p_{m}^{m}}$ and $G_{2}=C_{p^{n}}$ are two cyclic groups of respective orders $p^{m}$ and $p^{n}$ and are generated, respectively, by $\sigma_{1}$ and $\sigma_{2}$. We have $K[G] \cong R\left[G_{2}\right]$. As $R$ is a commutative ring and local and $G_{2}$ is a p-group, by [4, Proposition 10, page 239], $R\left[G_{2}\right]$ is a local ring. Therefore $K[G]$ is a local ring. As $K$ is commutative ring and local and $G$ is a $p$-group, $K[G]$ is a local ring by $[4$, Proposition 10, page 239]. So the $K[G]$-projective modules are free $K[G]$-modules.

Lemma 5. Let $M$ be a $K[G]$-module. Then, $M /\left(\sigma_{1}-1\right) M$ is a $K\left[G_{2}\right]$-module (also $M /\left(\sigma_{2}-1\right) M$ is a $K\left[G_{1}\right]$-module).

Proof. This lemma is a particular case of a more general result (see [5, page 386]). But for this particular case, we can give the following direct proof: $M /\left(\sigma_{1}-1\right) M$ is a $\left(K\left[G_{1}\right] /\left(\sigma_{1}-1\right) K\left[G_{1}\right]\right)\left[G_{2}\right]$-module, and we have already seen that $\left(\sigma_{1}-1\right) K\left[G_{1}\right]$ is the unique maximal ideal of $K\left[G_{1}\right]$ and $K\left[G_{1}\right] /\left(\sigma_{1}-1\right) K\left[G_{1}\right] \cong K$. So $M /\left(\sigma_{1}-1\right) M$ is a $K\left[G_{2}\right]-$ module.

Similarly we show that $M /\left(\sigma_{2}-1\right) M$ is a $K\left[G_{1}\right]$-module.

Proposition 6. Let $M$ be a free $K[G]$-module of rank $l$. Then, $M /\left(\sigma_{1}-1\right) M$ is a free $K\left[G_{2}\right]$-module and $M /\left(\sigma_{2}-1\right) M$ is a free $K\left[G_{1}\right]$-module of the same rank $l$. 
Proof. As $K[G]$ is a local ring, $K[G]$-projective modules are free $K[G]$-modules, and therefore this proposition is only a particular case of a more general result (see [5, Lemma 2.2.]). But for this particular case, we can give the following specific proof: we have $M \cong(K[G])^{l}=\left(K\left[G_{1} \times G_{2}\right]\right)^{l}$. So $M \cong\left(K\left[G_{1}\right]\left[G_{2}\right]\right)^{l}$. Then, we have

$$
\begin{aligned}
\left(\sigma_{1}-1\right) M & \cong\left(\sigma_{1}-1\right)\left(K\left[G_{1}\right]\left[G_{2}\right]\right)^{l} \\
& \cong\left(\left(\sigma_{1}-1\right) K\left[G_{1}\right]\left[G_{2}\right]\right)^{l} .
\end{aligned}
$$

Hence,

$$
\begin{aligned}
\frac{M}{\left(\sigma_{1}-1\right) M} & \cong \frac{\left(K\left[G_{1}\right]\left[G_{2}\right]\right)^{l}}{\left(\left(\sigma_{1}-1\right) K\left[G_{1}\right]\left[G_{2}\right]\right)^{l}} \\
& \cong\left(\frac{K\left[G_{1}\right]\left[G_{2}\right]}{\left(\sigma_{1}-1\right) K\left[G_{1}\right]\left[G_{2}\right]}\right)^{l} \\
& \cong\left(\frac{K\left[G_{1}\right]}{\left(\sigma_{1}-1\right) K\left[G_{1}\right]}\left[G_{2}\right]\right)^{l} .
\end{aligned}
$$

As $K\left[G_{1}\right] /\left(\sigma_{1}-1\right) K\left[G_{1}\right] \cong K$ (as we have already seen), $M /\left(\sigma_{1}-1\right) M \cong\left(K\left[G_{2}\right]\right)^{l}$. So $M /\left(\sigma_{1}-1\right) M$ is a free $K\left[G_{2}\right]-$ module of rank $l$.

Similarly we show that $M /\left(\sigma_{2}-1\right) M$ is a free $K\left[G_{1}\right]$ module of rank $l$.

Proposition 7. Let $M$ be a $K[G]$-module. If $M \downarrow_{G_{1}}$ is a free $K\left[G_{1}\right]$-module and $M /\left(\sigma_{1}-1\right) M$ is a free $K\left[G_{2}\right]$-module, then $M$ is a free $K[G]$-module.

Proof. $R=K\left[G_{1}\right]$ is a principal Artinian local ring with residue field $K$ and $\sigma_{1}-1$ is a generator of its maximal ideal. $M \downarrow_{G_{1}}$ is a free $R$-module and $Q=M /\left(\sigma_{1}-1\right) M$ is a projective $K\left[G_{2}\right]$-submodule of $M /\left(\sigma_{1}-1\right) M$. Then, $M=P \oplus M^{\prime}$, where $P$ is a projective $R\left[G_{2}\right]$-module and $P /\left(\sigma_{1}-1\right) P \cong Q=$ $M /\left(\sigma_{1}-1\right) M$ (according to [3, Proposition 4.13]). We have

$$
\begin{aligned}
\frac{M}{\left(\sigma_{1}-1\right) M} & \cong \frac{P \oplus M^{\prime}}{\left(\sigma_{1}-1\right)\left(P \oplus M^{\prime}\right)} \\
& \cong \frac{P \oplus M^{\prime}}{\left(\sigma_{1}-1\right) P \oplus\left(\sigma_{1}-1\right) M^{\prime}} \\
& \cong \frac{P}{\left(\sigma_{1}-1\right) P} \oplus \frac{M^{\prime}}{\left(\sigma_{1}-1\right) M^{\prime}} \\
& \cong \frac{M}{\left(\sigma_{1}-1\right) M} \oplus \frac{M^{\prime}}{\left(\sigma_{1}-1\right) M^{\prime}} .
\end{aligned}
$$

So $M^{\prime} /\left(\sigma_{1}-1\right) M^{\prime}=0$. By Nakayama's lemma and the remark following it, $M^{\prime}=0$. Therefore, $M=P$ which is projective $R\left[G_{2}\right]$-module. As $R\left[G_{2}\right] \cong K[G]$ is a local ring, $M$ is a free $K[G]$-module.

Let $J_{i}$ be the Jacobson radical of $K\left[G_{i}\right]$ for $i \in\{1,2\}$. Note that if $K$ is of characteristic $p$ (as here) and $G^{\prime}$ is a cyclic $p$ group, then the Jacobson radical of $K\left[G^{\prime}\right]$ is none other than $(\sigma-1) K\left[G^{\prime}\right]$, where $\sigma$ is a generator of $G^{\prime}$ (see [5, page 122]).
Let $M$ be a finitely generated $K[G]$-module and $k$ a natural number such that $1 \leq k \leq p^{n}$. As $K[G] \cong R\left[G_{2}\right], M$ is a $R\left[G_{2}\right]$-module. So $M / J_{1} M$ is a $K[X] /(X-1)^{p^{n}}$-module. $M$ is called of type $k$ if $M / J_{1} M$ is a free $K[X] /(X-1)^{k}$-module (terminology of [6]).

Lemma 8. If $M$ is a $K[G]$-module of type $k$ with $k=p^{r}$ and $0<r \leq n$ and $H_{2}$ is the subgroup of $G_{2}$ generated by $\sigma_{2}^{p^{n-r}}$, then $M / J_{1} M$ is a free $K\left[H_{2}\right]$-module.

Proof. As $M$ is of type $k, M / J_{1} M$ is a free $K[X] /(X-1)^{k}$ module. Define

$$
\begin{gathered}
\psi: \frac{K[X]}{(X-1)^{k}} \longrightarrow K\left[H_{2}\right], \\
\bar{X} \longmapsto \sigma_{2}^{p^{n-r}},
\end{gathered}
$$

where $\psi$ is a well-defined $K$-algebra homomorphism. It is not difficult to show that $\psi$ is an isomorphism (using an argument similar to that done in the proof of Lemma 3). So $M / J_{1} M$ is a free $\mathrm{K}\left[\mathrm{H}_{2}\right]$-module.

Theorem 9. Let $M$ be a $K[G]$-module of type $k$, with $J_{2}^{k} M=$ 0 , and let $\mathrm{H}_{2}$ be the subgroup of $\mathrm{G}_{2}$ generated by $\sigma_{2}^{p^{n-r}}$ with $0<r \leq n$. If $M \downarrow_{G_{1}}$ is $R$-free and $k=p^{r}$, then $M$ is a free $K\left[G_{1} \times H_{2}\right]$-module.

Proof. $M$ is an $R\left[G_{2}\right]$-module $R$-free. We have $J_{2}^{k} M=0$, so $\left(\sigma_{2}-1\right)^{k} M=0$, and therefore $\left(\left(\sigma_{2}-1\right)^{k} R\left[G_{2}\right]\right) M=0$. So $M$ is an $R\left[G_{2}\right] /\left(\sigma_{2}-1\right)^{k} R\left[G_{2}\right]$-module $R$-free. By Lemma 3, $R\left[G_{2}\right] /\left(\sigma_{2}-1\right)^{k} R\left[G_{2}\right] \cong R\left[H_{2}\right]$; then $M$ is an $R\left[H_{2}\right]$ module $R$-free. $M / J_{1} M$ is a free $K[X] /(X-1)^{k}$-module, so by Lemma 8 this is a free $K\left[H_{2}\right]$-module. In conclusion $M$ is a $K\left[G_{1} \times H_{2}\right]$-module such that

$$
\begin{aligned}
& M \downarrow_{G_{1}} \text { is a free } k\left[G_{1}\right] \text {-module, } \\
& \frac{M}{J_{1} M} \text { is a free } K\left[H_{2}\right] \text {-module. }
\end{aligned}
$$

So by Proposition $7 M$ is a free $K\left[G_{1} \times H_{2}\right]$-module.

In Theorem 9 we assumed that the $k[G]$-module $M$ satisfies the following condition: $M \downarrow_{G_{1}}$ is $R$-free. So it is useful to know when this condition is satisfied. This is the subject of the following result.

Theorem 10. Let $M$ be a $k[G]$-module and $\sigma$ an element of $G$ of order $p^{r}$. The following conditions are equivalent:

(1) $M \downarrow_{\langle\sigma\rangle}$ is free;

(2) $\operatorname{dim}_{k}(M)=\left(p^{r} /\left(p^{r}-1\right)\right) \operatorname{dim}_{k}((\sigma-1) M)$;

(3) $\operatorname{dim}_{k}(M)=\operatorname{dim}_{k}((\sigma-1) M)+\operatorname{dim}_{k}\left((\sigma-1)^{p^{r}-1} M\right)$.

Proof. (1) $\Rightarrow$ (2) Assume that $M \downarrow_{\langle\sigma\rangle}$ is free. There exists a nonzero natural number $n$ such that $M \downarrow_{\langle\sigma\rangle} \cong(k[\langle\sigma\rangle])^{n}$. The endomorphism $\varphi$ of $M$ defined by $\varphi(m)=(\sigma-1) m$ for all 
$m \in M$ is nilpotent of nilpotency index $p^{r}$, and $k[\langle\sigma\rangle]$ is an indecomposable $k[\langle\sigma\rangle]$-module. Therefore $M$ has a basis in which the matrix of $\varphi$ is a Jordan matrix. This matrix is formed of $n$ blocks of order $p^{r}$ all equal to

$$
\left(\begin{array}{cccc}
0 & 1 & & \\
& \ddots & \ddots & \\
& & \ddots & 1 \\
& & & 0
\end{array}\right)
$$

So $\operatorname{dim}_{k}(M)=p^{r} \times n$. We can easily see that $\operatorname{dim}_{k}((\sigma-1) M)=$ $n \times\left(p^{r}-1\right)$. Therefore $\operatorname{dim}_{k}(M)=\left(p^{r} /\left(p^{r}-1\right)\right) \operatorname{dim}_{k}((\sigma-1) M)$.

$(2) \Rightarrow$ (3) Now, assume that $\operatorname{dim}_{k}(M)=\left(p^{r} /\left(p^{r}-\right.\right.$ 1)) $\operatorname{dim}_{k}((\sigma-1) M)$. So $\operatorname{dim}_{k}(M)=p^{r} \times\left(\operatorname{dim}_{k}(M)-\operatorname{dim}_{k}((\sigma-\right.$ 1)M)). As $\operatorname{dim}_{k}(M)-\operatorname{dim}_{k}((\sigma-1) M)$ is the number of blocks of the Jordan matrix of $\varphi$, the order of each block is less than or equal to $p^{r}$, and $\operatorname{dim}_{k}(M)$ is equal to the sum of the orders of these blocks, then the order of each block is $p^{r}$. Therefore $\operatorname{dim}_{k}\left((\sigma-1)^{p^{r}-1} M\right)$ is equal to the number of Jordan blocks of $\varphi$. So $\operatorname{dim}_{k}(M)-\operatorname{dim}_{k}((\sigma-1) M)=\operatorname{dim}_{k}\left((\sigma-1)^{p^{r}-1} M\right)$; that is, $\operatorname{dim}_{k}(M)=\operatorname{dim}_{k}((\sigma-1) M)+\operatorname{dim}_{k}\left((\sigma-1)^{p^{r}-1} M\right)$.

(3) $\Rightarrow$ (1) Assume that $\operatorname{dim}_{k}(M)=\operatorname{dim}_{k}((\sigma-1) M)+$ $\operatorname{dim}_{k}\left((\sigma-1)^{p^{r}-1} M\right)$. So $\operatorname{dim}_{k}\left((\sigma-1)^{p^{r}-1} M\right)$ is equal to the number of Jordan blocks of $\varphi$. Therefore the order of each Jordan block is equal to $p^{r}$. So the modules contained in a decomposition of $M \downarrow_{\langle\sigma\rangle}$ as a direct sum of indecomposable modules are of the form $k[\langle\sigma\rangle]$; that is, $M \downarrow_{\langle\sigma\rangle}$ is free.

\section{Classification of Finitely \\ Generated $K\left[C_{p^{m}} \times C_{p^{n}}\right]$-Modules: Use of Module Extensions}

Let $G$ be a finite group and let $R$ be a ring. Let $M_{1}$ and $M_{2}$ be two $R[G]$-modules. We put $T=\operatorname{Hom}_{R}\left(M_{2}, M_{1}\right)$. $T$ has a natural structure as a $(R[G], R[G])$-bimodule centralized by $R$ (see [7, section 25]). Explicitly, we have

$$
\begin{array}{r}
(\lambda f) m_{2}=\lambda \cdot f\left(m_{2}\right), \quad(f \lambda) m_{2}=f\left(\lambda m_{2}\right) \\
\forall \lambda \in R[G], \quad m_{2} \in M_{2}, \quad f \in T .
\end{array}
$$

A derivation $F: R[G] \rightarrow T$ is an $R$-homomorphism satisfying

$$
F_{\lambda^{\prime} \lambda}=\lambda^{\prime} F_{\lambda}+F_{\lambda^{\prime}} \lambda \quad \forall \lambda, \lambda^{\prime} \in R[G] .
$$

Derivations from $R[G]$ into $T$ form an $R$-module $\operatorname{Der}(R[G], T)$. For $F \in \operatorname{Der}(R[G], T)$ we equip $M_{1} \times M_{2}$ with an $R[G]$-module structure by

$$
\begin{aligned}
& \lambda\left(m_{1}, m_{2}\right)=\left(\lambda m_{1}+F_{\lambda}\left(m_{2}\right), \lambda m_{2}\right) \\
& \forall\left(m_{1}, m_{2}\right) \in M_{1} \times M_{2}, \quad \lambda \in R[G] .
\end{aligned}
$$

This $R[G]$-module is denoted by $M_{1} \times_{F} M_{1}$ as in [8].

An extension of $M_{2}$ by $M_{1}$ is an $R[G]$-exact sequence $\mathrm{O} \rightarrow M_{1} \rightarrow X \rightarrow M_{2} \rightarrow O$. Let $O \rightarrow M_{1} \stackrel{u}{\rightarrow}$ $X \stackrel{v}{\rightarrow} M_{2} \rightarrow \mathrm{O}$ and $\mathrm{O} \rightarrow M_{1} \stackrel{u^{\prime}}{\rightarrow} X^{\prime} \stackrel{v^{\prime}}{\rightarrow} M_{2} \rightarrow O$ be a pair of extensions of $M_{2}$ by $M_{1}$. These two extensions are equivalent if there exists an isomorphism of $R[G]$-modules $\Phi: X \rightarrow X^{\prime}$ such that $\Phi$ ou $=u^{\prime}$ and $v^{\prime} o \Phi=v$. These equivalence classes of extensions form an $R$-module $\operatorname{Ext}_{R[G]}^{1}\left(M_{2}, M_{1}\right)$. The $R[G]$-modules sequence $O \rightarrow M_{1} \stackrel{i}{\rightarrow}$ $M_{1} \times_{F} M_{2} \stackrel{j}{\rightarrow} M_{2} \rightarrow O$, where $i$ and $j$ denote, respectively, the canonical injection from $M_{1}$ to $M_{1} \times_{F} M_{2}$ and the second projection from $M_{1} \times_{F} M_{2}$ to $M_{2}$, is exact. The equivalence class of this sequence is denoted by $\left[M_{1} \times_{F} M_{2}\right]$.

Remark 11. With the previous notations, derivations $F$ and modules $M_{1} \times_{F} M_{2}$ play the same role as the cocycles $\alpha$ and modules $M_{1} \times_{\alpha} M_{2}$ defined in [8].

From Proposition 25.10 of [7] we have the following result.

Proposition 12. The correspondence $\theta: \operatorname{Der}(R[G], T) \rightarrow$ $\operatorname{Ext}_{R[G]}^{1}\left(M_{2}, M_{1}\right)$ defined by $\theta(F)=\left[M_{1} \times_{F} M_{2}\right]$ is surjective whenever $M_{2}$ is finitely generated and projective as $R$-module.

From Theorems 5.2 and 5.3 of [9] we have the following result.

Proposition 13. Let $G$ be a cyclic group of order $p^{r}$ generated by an element $\sigma, K$ a field of characteristic $p$, and $M$ an indecomposable $K[G]$-module. Then, $M$ is isomorphic to ( $\sigma-$ $1)^{s} K[G]$, where $s$ is a natural number strictly less than $p^{r}$.

Lemma 14. Let $R$ be a ring and $G=G_{1} \times G_{2}$ a direct product of two finite groups. Let $M$ be an $R[G]$-module such that the action of $G_{1}$ on $M$ is trivial and let $M^{\prime}$ be an $R\left[G_{2}\right]$-module. If $M$ is isomorphic to $M^{\prime}$ as $R\left[G_{2}\right]$-modules and if we extend the action of $G_{2}$ on $M^{\prime}$ to $G$ by $\sigma \cdot m^{\prime}=m^{\prime}, \forall\left(\sigma, m^{\prime}\right) \in G_{1} \times M^{\prime}$, then $M$ is isomorphic to $M^{\prime}$ as $R[G]$-modules.

Proof. Let $\psi: M \rightarrow M^{\prime}$ be an isomorphism of $R\left[G_{2}\right]$ modules. We extend the action of $G_{2}$ on $M^{\prime}$ to $G$ by $\sigma \cdot m^{\prime}=$ $m^{\prime}, \forall\left(\sigma, m^{\prime}\right) \in G_{1} \times M^{\prime}$. We easily see that the application $\psi: M \rightarrow M^{\prime}$ is an isomorphism of $R[G]$-modules.

Let $K$ be a commutative field of characteristic $p>0$. Let $G=G_{1} \times G_{2}$, where $G_{1}=C_{p^{m}}$ and $G_{2}=C_{p^{n}}$ are two cyclic groups of respective orders $p^{m}$ and $p^{n}$ and are generated, respectively, by $\sigma_{1}$ and $\sigma_{2}$, and let $J_{1}$ be the Jacobson radical of $K\left[G_{1}\right]$.

Proposition 15. Let $M$ be a finitely generated $K[G]$-module. If $J_{1} M=0$, then there exists a nonzero natural number $n^{\prime}$ such that $M \cong \oplus_{i=1}^{n^{\prime}}\left(\sigma_{2}-1\right)^{k_{i}} K\left[G_{2}\right], 0 \leq k_{i}<p^{n}$, as $K[G]$-modules, where the action of $G_{1}$ on $\oplus_{i=1}^{n^{\prime}}\left(\sigma_{2}-1\right)^{k_{i}} K\left[G_{2}\right]$ is trivial.

Proof. If $J_{1} M=0$, then the action of $G_{1}$ on $M$ is trivial since $J_{1}=\left(\sigma_{1}-1\right) K\left[G_{1}\right]$. By Proposition 13 , there exists a nonzero natural number $n^{\prime}$ such that $M \cong \oplus_{i=1}^{n^{\prime}}\left(\sigma_{2}-1\right)^{k_{i}} K\left[G_{2}\right]$, $0 \leq k_{i}<p^{n}$, as $K\left[G_{2}\right]$-modules. Then, Lemma 14 allows concluding the following. 
Theorem 16. Let $M$ be a finitely generated $K[G]$-module. If $J_{1}^{2} M=0$, then there exist two nonzero natural numbers $n^{\prime}$ and $n^{\prime \prime}$ and two $K[G]$-modules $M_{1}=\oplus_{i=1}^{n^{\prime}}\left(\sigma_{2}-1\right)^{k_{i}} K\left[G_{2}\right], 0 \leq k_{i}<$ $p^{n}$, and $M_{2}=\oplus_{i=1}^{n^{\prime \prime}}\left(\sigma_{2}-1\right)^{k_{i}^{\prime}} K\left[G_{2}\right], 0 \leq k_{i}^{\prime}<p^{n}$, where the action of $G_{1}$ on $M_{1}$ and $M_{2}$ is trivial, and there is a derivation $F$ from $K[G]$ in $\operatorname{Hom}_{K}\left(M_{2}, M_{1}\right)$ such that $M \cong M_{1} \times_{F} M_{2}$.

Proof. We have the exact sequence of $K[G]$-modules $O \rightarrow$ $J_{1} M \hookrightarrow M \rightarrow M / J_{1} M \rightarrow O$. As $J_{1}^{2} M=0, J_{1}\left(J_{1} M\right)=0$. So by Proposition 15 there exists a nonzero natural number $n^{\prime}$ such that $J_{1} M \cong \oplus_{i=1}^{n^{\prime}}\left(\sigma_{2}-1\right)^{k_{i}} K\left[G_{2}\right], 0 \leq k_{i}<p^{n}$, as $K[G]$-modules, where the action of $G_{1}$ on $\oplus_{i=1}^{n^{\prime}}\left(\sigma_{2}-1\right)^{k_{i}} K\left[G_{2}\right]$ is trivial. We set $M_{1}=\oplus_{i=1}^{n^{\prime}}\left(\sigma_{2}-1\right)^{k_{i}} K\left[G_{2}\right]$. We have $J_{1}\left(M / J_{1} M\right)=0$. So by Proposition 15 there exists a nonzero natural number $n^{\prime \prime}$ such that $M / J_{1} M \cong \oplus_{i=1}^{n^{\prime \prime}}\left(\sigma_{2}-1\right)^{k_{i}^{\prime}} K\left[G_{2}\right]$, $0 \leq k_{i}^{\prime}<p^{n}$, as $K[G]$-modules, where the action of $G_{1}$ on $\oplus_{i=1}^{n^{\prime \prime}}\left(\sigma_{2}-1\right)^{k_{i}^{\prime}} K\left[G_{2}\right]$ is trivial. We set $M_{2}=\oplus_{i=1}^{n^{\prime \prime}}\left(\sigma_{2}-\right.$ $1)^{k_{i}^{\prime}} K\left[G_{2}\right]$. Then, Proposition 12 shows that $M \cong M_{1} \times_{F} M_{2}$ for a derivation $F$ from $K[G]$ in $\operatorname{Hom}_{K}\left(M_{2}, M_{1}\right)$.

If $p=2, G_{1}=C_{2}$, and $G_{2}=C_{2^{n}}$, then we have the following corollary.

Corollary 17. For all finitely generated $K[G]$-modules there exist two nonzero natural numbers $n^{\prime}$ and $n^{\prime \prime}$ and two $K[G]-$ modules $M_{1}=\oplus_{i=1}^{n^{\prime}}\left(\sigma_{2}-1\right)^{k_{i}} K\left[G_{2}\right], 0 \leq k_{i}<2^{n}$, and $M_{2}=\oplus_{i=1}^{n^{\prime \prime}}\left(\sigma_{2}-1\right)^{k_{i}^{\prime}} K\left[G_{2}\right], 0 \leq k_{i}^{\prime}<2^{n}$, where the action of $G_{1}$ on $M_{1}$ and $M_{2}$ is trivial, and there is a derivation $F$ from $K[G]$ in $\operatorname{Hom}_{K}\left(M_{2}, M_{1}\right)$ such that $M \cong M_{1} \times_{F} M_{2}$.

Proof. We have $J_{1}=\left(\sigma_{1}-1\right) K\left[G_{1}\right]$ and as $\left(\sigma_{1}-1\right)^{2}=0$ since the field $K$ is of characteristic $p=2, J_{1}^{2}=0$. So $J_{1}^{2} M=0$ for $K[G]$-module of finite type $M$. The rest is a simple application of Theorem 16.

Now we return to cases $G_{1}=C_{p^{m}}$ and $G_{2}=C_{p^{n}}$, where $G_{2}$ is generated by an element $\sigma_{2}$, and let $J_{2}$ be the Jacobson radical of $K\left[G_{2}\right]$. For an integer $k=p^{r}$ with $0<r \leq n$ and for the subgroup $\mathrm{H}_{2}$ of $G_{2}$ generated by $\sigma_{2}^{p^{n-r}}$, we have the following result.

Theorem 18. Let $M$ be a finitely generated $K[G]-$ module, with $J_{2}^{k+1} M=0$. If $M / J_{2}^{k} M \downarrow_{G_{1}}$ is $R$-free and of type $k$, then there exist two nonzero natural numbers $n^{\prime}$ and $n^{\prime \prime}$ and two $K[G]-$ modules $M_{1}=\oplus_{i=1}^{n^{\prime}}\left(\sigma_{1}-1\right)^{k_{i}} K\left[G_{1}\right], 0 \leq k_{i}<p^{n}$, and $M_{2}=$ $\left(K\left[G_{1} \times H_{2}\right]\right)^{n^{\prime \prime}}$, where the action of $G_{2}$ on $M_{1}$ is trivial, and there is a derivation $F$ from $K[G]$ in $\operatorname{Hom}_{K}\left(M_{2}, M_{1}\right)$ such that $M \cong M_{1} \times_{F} M_{2}$.

Proof. We have the following exact sequence:

$$
O \longrightarrow J_{2}^{k} M \hookrightarrow M \longrightarrow \frac{M}{J_{2}^{k} M} \longrightarrow O
$$

As $J_{2}\left(J_{2}^{k} M\right)=0$, by Proposition 15 , there exists a nonzero natural number $n^{\prime}$ such that $J_{2}^{k} M \simeq \oplus_{i=1}^{n^{\prime}}\left(\sigma_{1}-1\right)^{k_{i}} K\left[G_{1}\right]$,
$0 \leq k_{i}<p^{n}$, as $K[G]$-modules, where the action of $G_{2}$ on $\oplus_{i=1}^{n^{\prime}}\left(\sigma_{1}-1\right)^{k_{i}} K\left[G_{1}\right]$ is trivial. $M / J_{2}^{k} M$ is a $K[G]$-module of type $k$ with $J_{2}^{k}\left(M / J_{2}^{k} M\right)=0$, more $M / J_{2}^{k} M \downarrow_{G_{1}}$ is $R$-free, and $k=p^{r}$ with $0<r \leq n$. Then, Theorem 9 shows that $M / J_{2}^{k} M$ is a free $K\left[G_{1} \times H_{2}\right]$-module. Therefore there exists a nonzero natural number $n^{\prime \prime}$ such that $M / J_{2}^{k} M \simeq\left(K\left[G_{1} \times H_{2}\right]\right)^{n^{\prime \prime}}$. The rest is a simple application of Proposition 12.

\section{Conflict of Interests}

The authors declare that there is no conflict of interests regarding the publication of this paper.

\section{References}

[1] S. Ö. Kaptanoğlu, "Structure and detection theorems for $k\left[C_{2} \times C_{4}\right]$-modules," The Rendiconti del Seminario Matematico della Università di Padova, vol. 123, pp. 169-189, 2010.

[2] P. Webb, Finite Group Representations for the Pure Mathematician, University of Minnesota, 2013.

[3] J. Thévenaz, Représentations linéaires de groupes finis en caractéristique $\mathrm{p}^{r}$, Université de Genève, 1980.

[4] K. W. Gruenberg, Cohomological Topics in Group Theory, vol. 143 of Lecture Notes in Mathematics, Springer, Berlin, Germany, 1970.

[5] G. Karpilovsky, The Jacobson Radical of Group Algebras, Notas De Mathematica, vol. 135, Elsevier Science, New York, NY, USA, 1987.

[6] J. Thévenaz, "Representations of finite groups in characteristic $p^{r}$, , Journal of Algebra, vol. 72, no. 2, pp. 478-500, 1981.

[7] C. W. Curtis and I. Reiner, Methods of Representation Theory, Wiley-Interscience, New York, NY, USA, 1981.

[8] M. E. Charkani and S. Bouhamidi, "Modular representations of Loewy length two," International Journal of Mathematics and Mathematical Sciences, no. 70, pp. 4399-4408, 2003.

[9] B. Huppert and N. Blackburn, Finite Groups II, Springer, Berlin, Germany, 1982. 


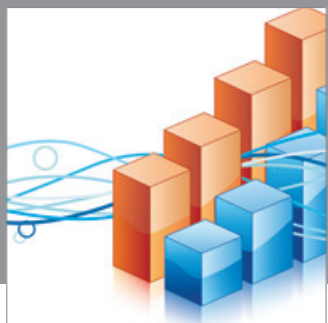

Advances in

Operations Research

mansans

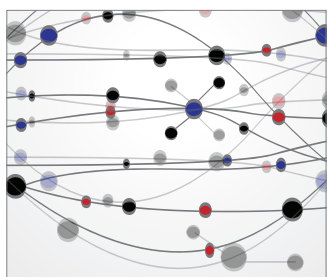

The Scientific World Journal
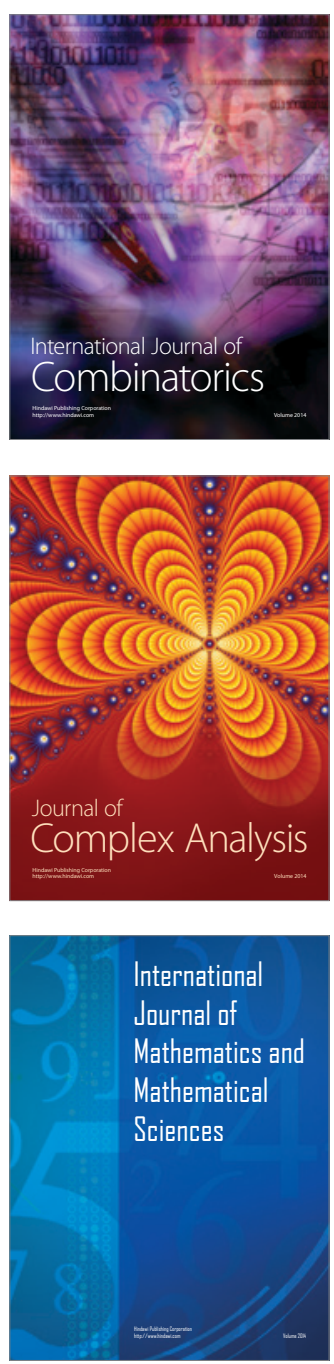
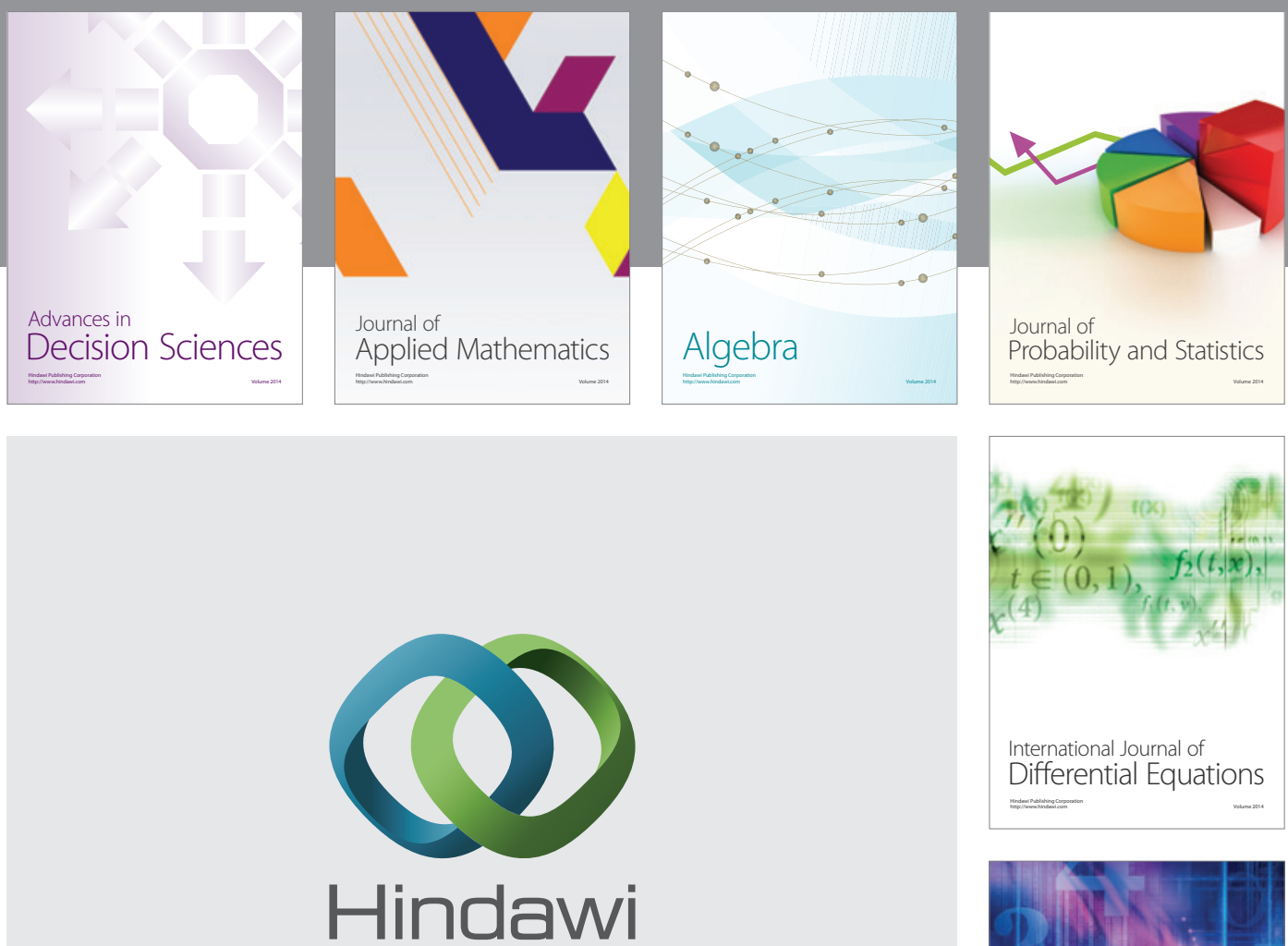

Submit your manuscripts at http://www.hindawi.com
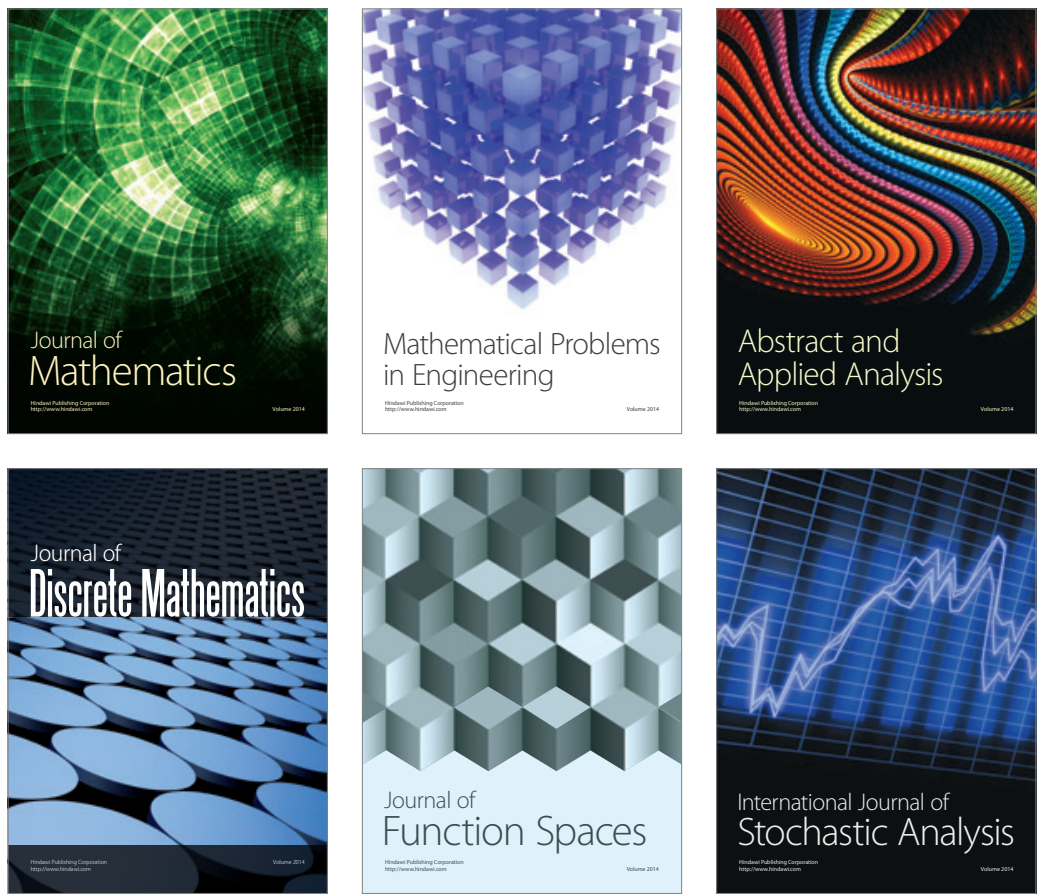

Journal of

Function Spaces

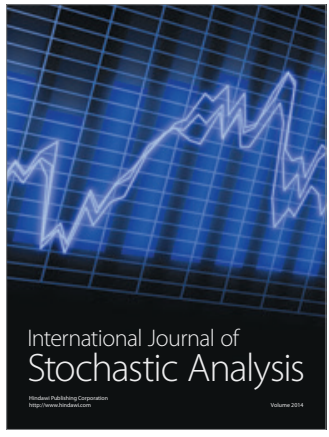

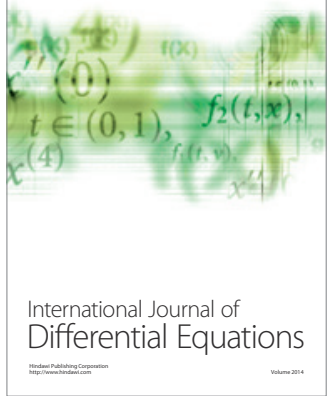
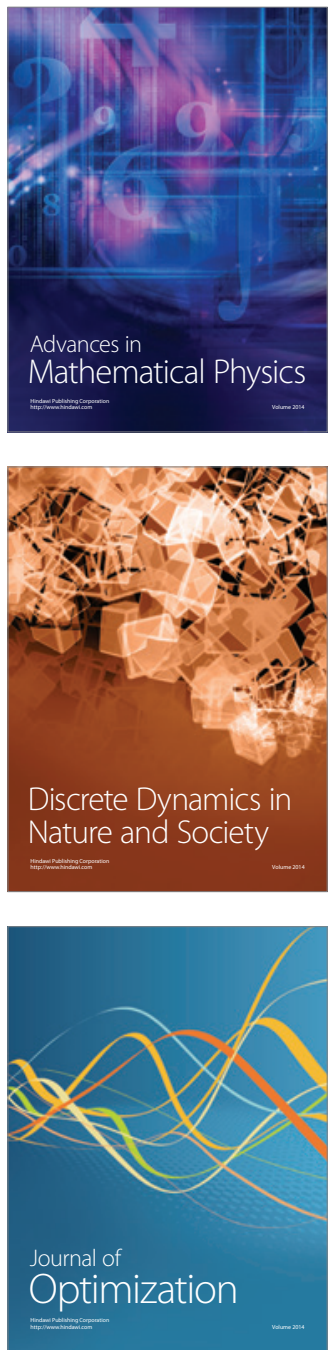